\title{
Search for neutrinoless double-beta decays in Ge-76 in the LEGEND experiment
}

\section{Jordan Myslik* for the LEGEND Collaboration}

Nuclear Science Division, Lawrence Berkeley National Laboratory, Berkeley, CA, USA

E-mail: jwmyslikelbl.gov

\begin{abstract}
The search for neutrinoless double-beta decay is the most sensitive technique to establish the Majorana nature of neutrinos. Two operating experiments that look for such decays in ${ }^{76} \mathrm{Ge}-$ GERDA and the MAJORANA DEMONSTRATOR - have achieved the lowest backgrounds and the best energy resolution in the signal region. These are two of the most important detector characteristics for sensitive searches of this undiscovered decay. The Large Enriched Germanium Experiment for Neutrinoless Double Beta Decay (LEGEND) Collaboration has formed to pursue a tonne-scale ${ }^{76} \mathrm{Ge}$ experiment that integrates the best technologies from these two experiments and others in the field. The Collaboration is developing a phased experimental program that uses existing resources as appropriate to expedite physics results, with the ultimate discovery potential at a decay half-life beyond $10^{28}$ years. In these proceedings, we will present the physics case, $R \& D$ efforts and implementation strategies of the LEGEND experiment.
\end{abstract}

The 39th International Conference on High Energy Physics (ICHEP2018)

4-11 July, 2018

Seoul, Korea

${ }^{*}$ Speaker. 


\section{Introduction}

Neutrinoless double-beta decay violates lepton number conservation by two units, and is a key feature of multiple neutrino mass generation models. It would be observed as a peak at the Q-value of an isotope's double-beta decay spectrum. For ${ }^{76} \mathrm{Ge}$, sensitivity to a half-life $>10^{28} \mathrm{y}$ in the light neutrino exchange model [1] would probe the entire neutrino mass inverted ordering (IO) parameter space and most of the normal ordering (NO) parameter space [2], making it a reasonable target for a future experiment. With a signal of only $\sim 0.5$ counts/t.y at a half-life of $10^{28} \mathrm{y}$, ultra-low backgrounds, a large fiducial mass, and a long counting time are required.

The Majorana Demonstrator [3] and GERDA (the GERmanium Detector Array) [4] are the two currently operating experiments using germanium crystal detectors enriched to $\sim 88 \%$ in ${ }^{76} \mathrm{Ge}(29.7 \mathrm{~kg}$ for the MAJORAna Demonstrator and $37.6 \mathrm{~kg}$ for GERDA). Both are located underground to reduce cosmic ray muon flux, at the Sanford Underground Research Facility (SURF) and the Laboratori Nazionali del Gran Sasso (LNGS), respectively. The MAJORANA DEMONSTRATOR's approach of two vacuum cryostats within a passive shield, with emphasis on ultra-clean components, is different from GERDA's approach of submerging their detectors in a liquid argon active shield. These approaches both resulted in lower backgrounds and better energy resolution than all other neutrinoless double-beta decay experiment technologies (GERDA holding the background record of $\sim 3$ counts/(FWHM.t.y), and the MAJORANA DEMONSTRATOR holding the energy resolution record of $2.5 \mathrm{keV}$ FWHM at the 2039-keV Q-value).

\section{LEGEND}

Using lessons learned from GERDA, the MAJORAnA DEMONSTRATOR, and contributions from other groups, the LEGEND (Large Enriched Germanium Experiment for Neutrinoless $\beta \beta$ Decay) collaboration plans to develop a ${ }^{76} \mathrm{Ge}$-based double-beta decay experimental program, proceeding in phases to a discovery potential at a half-life beyond $10^{28}$ years. Existing resources will be used as appropriate to expedite physics results.

The 200-kg first phase, LEGEND-200, will modify existing GERDA infrastructure at LNGS, permitting early science (physics data expected in 2021) with a world-leading experiment (sensitivity greater than $10^{27}$ years with $1 \mathrm{t} \cdot \mathrm{y}$ of exposure). Between GERDA, the MAJORANA DEMONSTRATOR, and dedicated test stands, reducing backgrounds to the goal of less than 0.6 counts/(FWHM.t.y) has already been demonstrated as feasible.

New infrastructure will house the subsequent stages up to the $1000 \mathrm{~kg}$ LEGEND-1000, over a timeline connected to the U.S. Department of Energy down-select process for the next generation neutrinoless double-beta decay experiment. To achieve a discovery potential at a halflife greater than $10^{28}$ years with approximately $10 \mathrm{t} \cdot \mathrm{y}$ of exposure, a background rate less than 0.1 counts/(FWHM.t.y) is required. The depth required for sufficiently low cosmogenic backgrounds (e.g. ${ }^{77 \mathrm{~m}} \mathrm{Ge}$ ) will impact the site choice, and is under investigation (e.g. [5]).

\section{Background reduction techniques}

A selection of the techniques to be used to achieve the LEGEND-200 and LEGEND-1000 background reduction goals are discussed in this section. 


\subsection{Electroformed copper}

For the most background-sensitive structural components (those closest to the detectors e.g. mounts, inner shield) the MAJORANA DEMONSTRATOR uses copper electroformed underground at Pacific Northwest National Laboratory and SURF, which achieved average U and Th background rates of $\leq 0.1 \mu \mathrm{Bq} / \mathrm{kg}$ each [6]. Production underground reduces backgrounds from cosmogenic ${ }^{60} \mathrm{Co}$. Electroformed copper should improve on GERDA radiopurity for LEGEND-200, and $37 \mathrm{~kg}$ currently in production at SURF is expected to be complete by the fall of 2019.

\subsection{Liquid argon veto}

External backgrounds are tagged using scintillation light they produce in the GERDA liquid argon (LAr) volume [7]. It is read out by photomultiplier tubes above and below the array, and a wavelength-shifting-fiber shroud surrounding the array, read out by silicon photomultipliers. An additional shroud around the central column was recently added to increase light collection efficiency. LEGEND-200 will use a similar design, though the optimal fibre geometry for light collection efficiency is under study. Improved LAr purity and Xe doping are being studied to improve light yield and attenuation. More radiopure fibres and signal amplification or digitization in LAr are also being studied.

The ${ }^{42} \mathrm{Ar}$ present in natural $\mathrm{Ar}$ is a background to consider, since it decays to a ${ }^{42} \mathrm{~K}$ ion which drifts to detectors, and beta decays to ${ }^{42} \mathrm{Ca}$ with $\mathrm{Q}=3.5 \mathrm{MeV}$. Nylon shrouds around the columns limit ${ }^{42} \mathrm{~K}$ drift in GERDA and the future LEGEND-200, and this background is cut with $99 \%$ efficiency by pulse shape analysis. For LEGEND-1000 this background could be removed completely by separating the detectors into four volumes containing underground $\mathrm{Ar}$ (free of ${ }^{42} \mathrm{Ar}$ ) separated by copper walls from a larger natural Ar volume. This design would require 21 tons $\left(15 \mathrm{~m}^{3}\right)$ of underground argon, a similar need to Darkside-20k [8].

\subsection{Front-end electronics}

The resistive feedback charge sensitive preamplifier for each MAJORAnA DEMONSTRATOR detector consists of the Low-Mass Front-End (LMFE) [9] close to each detector's p+ contact (to minimize noise), and the warm preamplifier outside the shield. An MX-11 JFET, the feedback resistor, and the feedback capacitor are on the LMFE, so the feedback loop runs $2.15 \mathrm{~m}$ out to the warm preamplifier and back. Backgrounds due to the LMFE are kept low (it is the most radiopure front-end in existence) through its low-mass design and selection of ultra-clean materials (e.g. the $400 \mathrm{~nm}$ sputtered amorphous Ge feedback resistor). The LMFE is in the baseline design for LEGEND-200, with research and development into its performance in LAr and with longer cables ongoing. Research and development into an ASIC preamplifier that could be placed near each LEGEND-1000 detector is also underway.

\subsection{Larger detectors}

Using larger detectors than the Majorana Demonstrator (P-type Point Contact detectors - PPCs - average $0.85 \mathrm{~kg}$ ) and GERDA (Broad Energy Germanium detectors - BEGes average $0.66 \mathrm{~kg}$ ) would reduce the surface to volume ratio, and require fewer cables and preamplifier front-ends per unit mass, reducing backgrounds. A new detector geometry, Inverted-Coaxial 
Point Contact (ICPC) detectors [10], have similar performance to PPCs and BEGes (excellent energy resolution and pulse-shape sensitivity) [11], but can be much larger. The current baseline for enriched detectors is $1.5-2.0 \mathrm{~kg}$, with detectors around $3 \mathrm{~kg}$ and up to $6 \mathrm{~kg}$ being investigated. GERDA deployed 5 enriched ICPC detectors (each $\sim 1.9 \mathrm{~kg}$ ) in a recent upgrade.

\section{Conclusions}

The Majorana Demonstrator and GERDA have demonstrated the lowest backgrounds and best energy resolution of all neutrinoless double-beta decay searches, but the next generation of experiments requires additional mass and further reduced backgrounds. Taking the best of these experiments, along with additional R\&D, LEGEND will proceed in a phased fashion towards a tonne-scale experiment with neutrinoless double-beta decay discovery potential at a half-life beyond $10^{28}$ years. The 200-kg first phase, LEGEND-200, has a background reduction goal that is already demonstrated to be feasible, and will begin taking data in 2021 .

\section{References}

[1] W. Rodejohann, Neutrino-less Double Beta Decay and Particle Physics, Int. J. Mod. Phys. E20 (2011) 1833 [1106.1334].

[2] M. Agostini, G. Benato and J. Detwiler, Discovery probability of next-generation neutrinoless double- $\beta$ decay experiments, Phys. Rev. D96 (2017) 053001 [1705. 02996].

[3] Majorana collaboration, N. Abgrall et al., The Majorana Demonstrator Neutrinoless Double-Beta Decay Experiment, Adv. High Energy Phys. 2014 (2014) 365432 [1308 . 1633].

[4] GERDA collaboration, K. H. Ackermann et al., The GERDA experiment for the search of $0 \mathrm{v} \beta \beta$ decay in ${ }^{76}$ Ge, Eur. Phys. J. C73 (2013) 2330 [1212 . 4067 ].

[5] C. Wiesinger, L. Pandola and S. Schönert, Virtual depth by active background suppression: Revisiting the cosmic muon induced background of GERDA Phase II, Eur. Phys. J. C78 (2018) 597 [1802.05040].

[6] N. Abgrall et al., The Majorana Demonstrator radioassay program, Nucl. Instrum. Meth. A828 (2016) 22 [1601.03779].

[7] GERDA collaboration, M. Agostini et al., Upgrade for Phase II of the Gerda experiment, Eur. Phys. J. C78 (2018) 388 [1711.01452].

[8] C. E. Aalseth et al., DarkSide-20k: A 20 tonne two-phase LAr TPC for direct dark matter detection at LNGS, Eur. Phys. J. Plus 133 (2018) 131 [1707.08145].

[9] N. Abgrall et al., The Majorana Low-noise Low-background Front-end Electronics, Phys. Procedia 61 (2015) 654 .

[10] R. J. Cooper, D. C. Radford, P. A. Hausladen and K. Lagergren, A novel HPGe detector for gamma-ray tracking and imaging, Nuclear Instruments and Methods in Physics Research A 665 (2011) 25 .

[11] A. Domula, M. Hult, Y. Kermaidic, G. Marissens, B. Schwingenheuer, T. Wester et al., Pulse shape discrimination performance of Inverted Coaxial Ge detectors, Nucl. Instrum. Meth. A891 (2018) 106 [1711.01433]. 